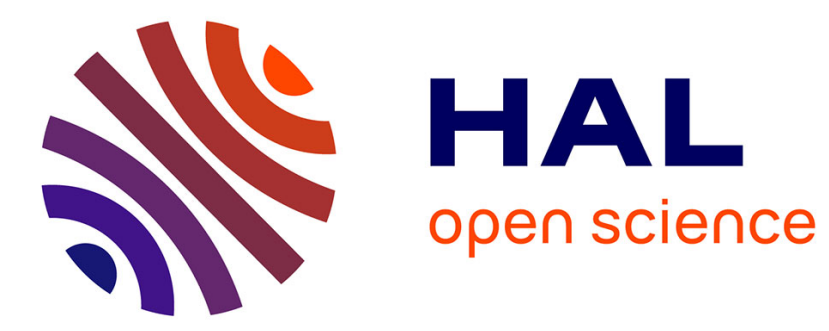

\title{
On space and time correlations of isotropic and rotating turbulence
}

\author{
Benjamin Favier, Fabien Godeferd, Claude Cambon
}

\section{To cite this version:}

Benjamin Favier, Fabien Godeferd, Claude Cambon. On space and time correlations of isotropic and rotating turbulence. Physics of Fluids, 2010, 22, pp.015101. 10.1063/1.3276290 . hal-00496218

\section{HAL Id: hal-00496218 \\ https://hal.science/hal-00496218}

Submitted on 30 Jun 2010

HAL is a multi-disciplinary open access archive for the deposit and dissemination of scientific research documents, whether they are published or not. The documents may come from teaching and research institutions in France or abroad, or from public or private research centers.
L'archive ouverte pluridisciplinaire HAL, est destinée au dépôt et à la diffusion de documents scientifiques de niveau recherche, publiés ou non, émanant des établissements d'enseignement et de recherche français ou étrangers, des laboratoires publics ou privés. 


\title{
On space and time correlations of isotropic and rotating turbulence
}

\author{
B. FAVIER ${ }^{1}$, F.S. GODEFERD, and C. CAMBON \\ Laboratoire de Mécanique des Fluides et d'Acoustique, UMR 5509, \\ École Centrale de Lyon, CNRS, UCBL, INSA - Université de Lyon \\ F-69134 Ecully Cedex, France \\ PACS: 47.27.ek ; 47.27.Gs ; 47.27.Sd
}

\begin{abstract}
Two different approaches are used for evaluating two-time correlations of Fourier velocity modes in isotropic, and in rotating, homogeneous incompressible turbulence. A synthetic model is proposed and compared with the results of direct numerical simulations. This kinematic model presents the advantage to incorporate different sources of unsteadiness, from arbitrary timescales to linear dynamics of rotating flows. The two main timescales characterizing the various processes involved in the dynamics of isotropic turbulence can be included: the sweeping effect, in which small scales of the flow are advected by the large scale motion, and the straining hypothesis-also called Kolmogorov or eddy turnover timescale. In the rotating case, the dispersion relation of inertial waves can also be included. Using Lighthill's theory, the effect of these different timescales on the sound emitted by such a model is also examined, and compared with direct numerical simulations.
\end{abstract}

\section{Introduction}

Space and time correlations of developed turbulent flows are of interest in many applications. Two-time two-point velocity correlations can be used for evaluating displacements of fluid particles [1]. In aeroacoustics, the computation of the sound radiated by isotropic turbulence depends strongly on the accurate estimation of the two-point and two-time

\footnotetext{
${ }^{1}$ corresponding author: benjamin.favier@ec-lyon.fr
} 
velocity correlations $[2,3]$. The small-scale fluctuations are spatially described in a good approximation by Kolmogorov theory [4], i.e. $\left\langle(u(\boldsymbol{x}+\boldsymbol{r})-u(\boldsymbol{r}))^{2}>\approx r^{2 / 3}\right.$. It is also possible to derive the frequency spectrum of the Lagrangian velocity field from local similarity arguments. However, this approach does not predict the correct behavior of the Lagrangian frequency spectrum. The sweeping effect is assumed to play an important role, and the mean dissipation $\epsilon$ is not the only parameter of interest in the dimensional analysis, so is the rms velocity $u_{0}$. Two distinct features can therefore be responsible for the temporal decorrelation of a Eulerian velocity field: the sweeping effect in which the dissipative eddies of the flow are advected by the large scale motion, and the Kolmogorov timescale based on local similarity arguments (also referred to as straining hypothesis or eddy-turnover timescale). These two hypotheses have been discussed for a long time $[5,6,7]$, and are assumed to be the two main timescales characterizing the various processes involved in the dynamics [8]. Some evidence of the sweeping effect in the inertial range can be found in both theoretical [7], experimental [9] and numerical [10] studies. Note however that the inertial eddy-turnover time has been observed in [11] as the dominant timescale.

This paper focuses on Eulerian two-time correlations of velocity Fourier modes, and more specifically on the sweeping and straining hypotheses. This field has already been studied using large-eddy simulation (LES) [12], as well as in the context of sound generation by isotropic turbulence [13]. In these cases, comparisons are made using LES, direct numerical simulations (DNS) and theoretical approaches. Since the sweeping effect is assumed to be part of the non-linear dynamics of the Navier-Stokes equations, it can be discussed using both numerical (such as LES and DNS) and theoretical approaches based on these equations. However, few numerical models offer the possibility to explicitly control the sources of unsteadiness, so that it is possible to look at their respective influences on time correlations.

The first aim of this paper is to propose an efficient numerical model in which both the straining and sweeping hypotheses can be introduced, and to look at their impact on 
space-time correlations. Kinematic simulation (KS) is such a model, since the unsteadiness is controlled at all scales of the flow. The modeling of these correlations is essential in many applications, e.g. as here rotating turbulence or the acoustic emission of turbulence. In the case of isotropic turbulence, analytical models for acoustic emission have been proposed $[13,14]$, which we complete in the present work with numerical results drawn from kinematic simulations and direct numerical simulations.

We also investigate two-time correlations of flows dominated by mean rotation. In rotating fluids, the linear solutions for two-point two-time velocity correlation can be used for estimating Lagrangian statistics [1]. KS has already been used in that context [15] since it is possible to explicitly include the linear dynamics of rotating fluids in the model. We are therefore also interested in the comparison of the two-time correlation of rotating turbulence computed using DNS and KS including linear dynamics.

These correlations were also numerically studied by [16] for sheared homogeneous turbulence. The impact of the mean shear flow, with rate $S$, on turbulence is thus different from the one of the solid body rotation, even if they both call into play an additional time scale: $S^{-1}$ versus $(2 \Omega)^{-1}$. The mean velocity gradient of the plane shear can be split into a symmetric part, related to an irrotational strain (rate $S / 2$ ), and an antisymmetric part, related to a mean vorticity of magnitude $S$ in the spanwise direction. These two parts have a different effect: the pure straining process deforms turbulent eddies directly, whereas solid body rotation results in dispersive waves. It alters the two-time second-order correlations (relevant here) and the energy cascade mediated by third-order velocity correlations (outside our scope, see e.g.[17]), without direct influence on single-time second-order correlations. As a consequence, the energy spectrum is directly affected by the mean shear, as shown in Rapid Distortion Theory (RDT), but not by pure rotation (see, e.g. [18]).

The following section will present the two numerical methods used in this paper: KS and DNS of forced turbulence. DNS is used to compute time correlations of velocity Fourier modes, and also provides the spectrum used to initialize KS. Section 3 is devoted 
to the comparison of space-time correlations and their Fourier transforms computed using DNS and KS in the isotropic case whereas section 4 is focused on the rotating case. An application to aeroacoustics, based on Lighthill's theory [19] and works by Rubinstein and Zhou [14], is finally presented in Section 5 .

\section{Numerical methods}

We compute two-time correlations and the sound generation of isotropic and rotating homogeneous steady turbulence. These quantities are derived from a velocity field which is generated using two different methods: DNS of the Navier-Stokes equations, that provide a fully non linearly evolving velocity field, and KS, which is based on a linear superposition of random Fourier modes. We describe each of the numerical procedures in the following, which are essentially the same as in [20].

\subsection{Direct Numerical Simulation}

A pseudo-spectral method is used to solve the incompressible Navier-Stokes equations in the rotating frame

$$
\begin{aligned}
\frac{\partial \boldsymbol{u}}{\partial t}+(\boldsymbol{u} \cdot \nabla) \boldsymbol{u}+2 \boldsymbol{\Omega} \times \boldsymbol{u} & =-\frac{1}{\rho_{0}} \nabla p+\nu \nabla^{2} \boldsymbol{u} \\
\nabla \cdot \boldsymbol{u} & =0
\end{aligned}
$$

where $\boldsymbol{u}(\boldsymbol{x}, t)$ is the fluctuating velocity field, $p$ the pressure, $\rho_{0}$ the density and $\nu$ the kinematic viscosity. $\Omega=|\Omega|$ is the rotation rate and the rotation axis is assumed to be vertical along the $z$ axis. The velocity field is computed in a cubic box of side $2 \pi$ with periodic boundary conditions using $256^{3}$ Fourier modes. The minimum wave number

is then $k_{\min }=1$. The initial incompressible velocity field is a random superposition of Fourier modes with an energy distribution given by the narrow-band spectrum $E(k, t=$ $0) \approx k^{4} \exp \left(-2\left(k / k_{i}\right)^{2}\right)[21]$. A spherical truncation of Fourier components is used to 


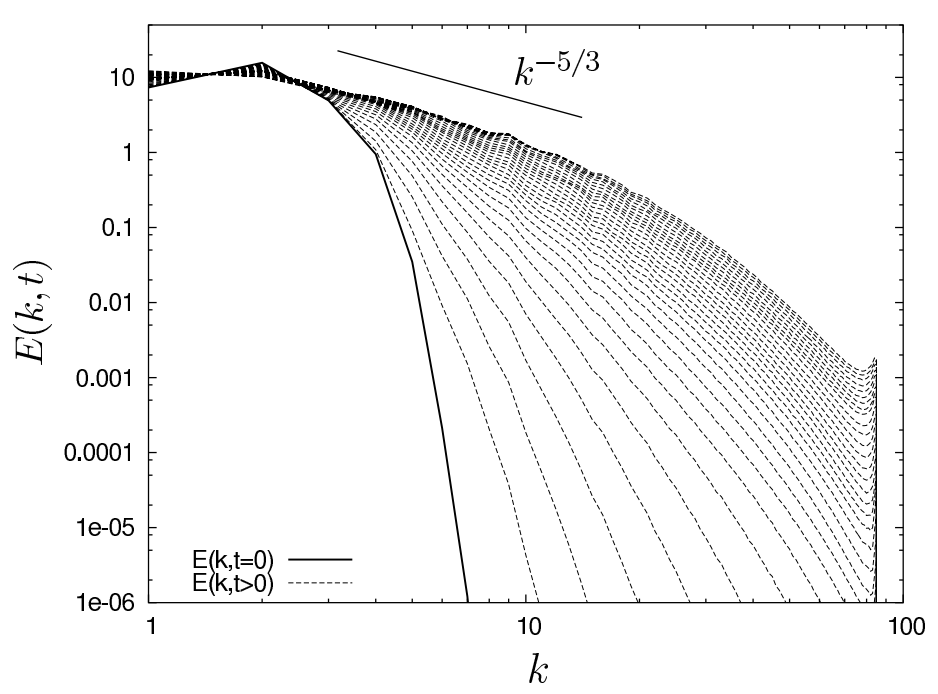

Figure 1: Direct Numerical Simulation of forced isotropic turbulence: Energy spectrum for different iterations in time, starting from an initial narrow-band analytical spectrum.

avoid aliasing and the time scheme is a third-order Adams-Bashforth. Since the viscous decay is discarded in KS, we need to force the turbulence in DNS to allow comparisons between the two models. Moreover, the acoustic emission of homogeneous turbulence is very sensitive to the rms velocity of the flow so that the forcing will be necessary in section 5, devoted to aeroacoustics. A quasi-steady state is reached after a few eddy turnover times by injecting the energy $\Delta E_{T}$ globally lost during one time step in an appropriately chosen range of wave numbers $\left[k_{1}, k_{2}\right]$. The Fourier components $\hat{u}_{i}(k, t)$ are renormalized with the coefficient

$$
\begin{gathered}
\beta=\sqrt{1+\frac{\Delta E_{T}}{\int_{k_{1}}^{k_{2} E(k) \mathrm{d} k}}} \text { if } k \in\left[k_{1}, k_{2}\right] \\
\text { otherwise. }
\end{gathered}
$$

Thus, the kinetic energy remains constant in both models. Figure 1 shows the corresponding evolution of the DNS energy spectrum in time, from the narrow band initial condition to a quasi-steady profile. The forced range of wave numbers is $\left[k_{1}=1, k_{2}=4\right]$. The impact of the forcing on time correlations and the smooth evolution in time of $\beta$ can be found in [20]. It is shown that the forcing method does not modify time correlations, except for forced modes whose correlation time is slightly larger than in the decaying case. 


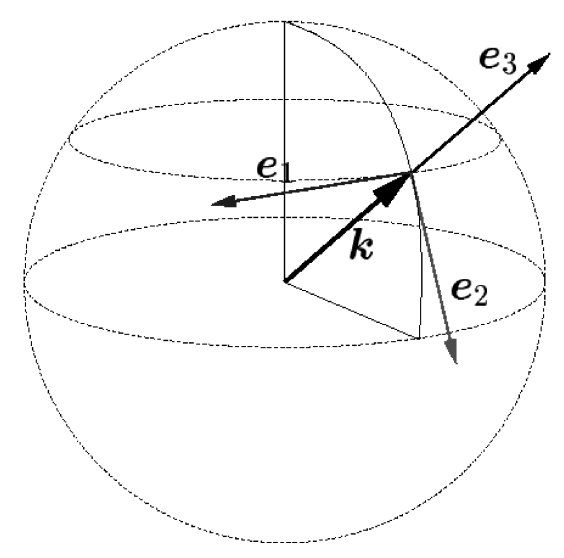

Figure 2: In the local Craya-Herring frame, a Fourier velocity vector $\hat{\boldsymbol{u}}(\boldsymbol{k})$ can be decomposed into its toroidal part $\hat{u}^{(1)}(\boldsymbol{k}) \boldsymbol{e}_{1}(\boldsymbol{k})$ and poloidal part $\hat{u}^{(2)}(\boldsymbol{k}) \boldsymbol{e}_{2}(\boldsymbol{k})$.

\section{$2.2 \quad$ Kinematic Simulation}

Originally, KS was used to compute single-particle diffusion of an isotropic velocity field [22]. Basically, the velocity field is a linear superposition of planar Fourier modes. As pointed out by Kraichnan, the main advantage of this model is to synthesize the velocity only among the particle trajectories. Since then, KS has been used in many applications, from Lagrangian diffusion of fluid particles $[23,24,25,26]$ to the propagation of acoustic waves through turbulence [27] and the noise modeling of turbulent jets [28]. Moreover, KS provides good two-time and two-point correlations compared to the ones obtained from DNS, at a reasonable numerical costs. Finally, background rotation or stratification can be introduced in KS through linear dynamics $[15,29]$. In the following, we present the basics of the model (for more details, see [30]), as well as some proposed improvements.

A fluctuating velocity vector is synthesized at any position $\boldsymbol{x}$ and at any time $t$ in the form

$$
\boldsymbol{u}(\boldsymbol{x}, t)=\Re \sum_{n=1}^{M}\left(u^{(1)}\left(\boldsymbol{k}_{n}\right) \boldsymbol{e}_{1}\left(\boldsymbol{k}_{n}\right)+u^{(2)}\left(\boldsymbol{k}_{n}\right) \boldsymbol{e}_{2}\left(\boldsymbol{k}_{n}\right)\right) e^{j\left(\boldsymbol{k}_{n} \cdot \boldsymbol{x}+\omega_{n} t\right)}
$$

where $\Re$ denotes the real part, $M$ the number of modes and $j^{2}=-1$. Many realizations of the flow (typically from 10 to 1000 realizations) and many modes (typically $N \approx 1000$ ) can be needed for statistical convergence. The incompressibility constraint $\boldsymbol{k} \cdot \hat{\boldsymbol{u}}(\boldsymbol{k})=$ 
0 is verified by construction since the two Fourier components $u^{(1)}\left(\boldsymbol{k}_{n}\right)$ and $u^{(2)}\left(\boldsymbol{k}_{n}\right)$ are located in a plane perpendicular to the local wave vector $\boldsymbol{k}_{n}$, i.e. in the plane $\left(\boldsymbol{e}_{1}\left(\boldsymbol{k}_{n}\right), \boldsymbol{e}_{2}\left(\boldsymbol{k}_{n}\right)\right)$, see figure 2. The amplitude of each Fourier mode is derived from an imposed isotropic energy spectrum $E(k)$, which has an analytical form in most studies. In our case, the quasi-steady spectrum computed from DNS is used as an input in KS so that the two numerical methods are quantitatively comparable. Note that, looking at equation (4), there is no viscous decay in the present version of our KS. However, since the spectrum is characterized by an integral scale $l_{0} \approx 1 / k_{i}$ where $k_{i}$ is the wave number of maximum energy $\left(k_{i} \approx 3\right.$ here), and a dissipative scale $\eta_{0} \approx 1 / k_{\eta}$, where $k_{\eta}$ is the Kolmogorov wave number, it is possible to derive a Reynolds number from KS as $R e \approx\left(l_{0} / \eta_{0}\right)^{3 / 4}$. The computational cost of KS does not depend on the choice of this Reynolds number so that it is possible to compute velocity fields with an arbitrary large inertial range. The limit in the present study is the resolution imposed by DNS.

In our KS model, the distribution of the $M$ wave vectors is different from the one used in the classical method, in which the wave number space is discretized with a geometrical distribution [30]. We introduce a more general statistical method to generate the wave vectors, which provides a sounder discretization than the classical method in the anisotropic cases. The wave number is considered as a random variable. Each Fourier modes carries the same amount of energy so that the pdf $P\left(k_{n}\right)$ of this variable can be calculated from

$$
P\left(k_{n}\right)=\frac{\int_{0}^{k_{n}} E(\kappa) \mathrm{d} \kappa}{\int_{0}^{\infty} E(\kappa) \mathrm{d} \kappa}
$$

where $E(k)$ is the prescribed energy spectrum. The wave vectors are built using this distribution and by introducing two random angles with isotropic distributions. Figure 3 presents the quasi-steady spectrum obtained by DNS, and the equivalent KS energy spectrum derived from it. The spatial correlations of KS are then directly linked to the prescribed spectrum. Note that, in this model, only the second order spectra may be explicitly included. Therefore higher-order spectra vanish. In some previous works, 


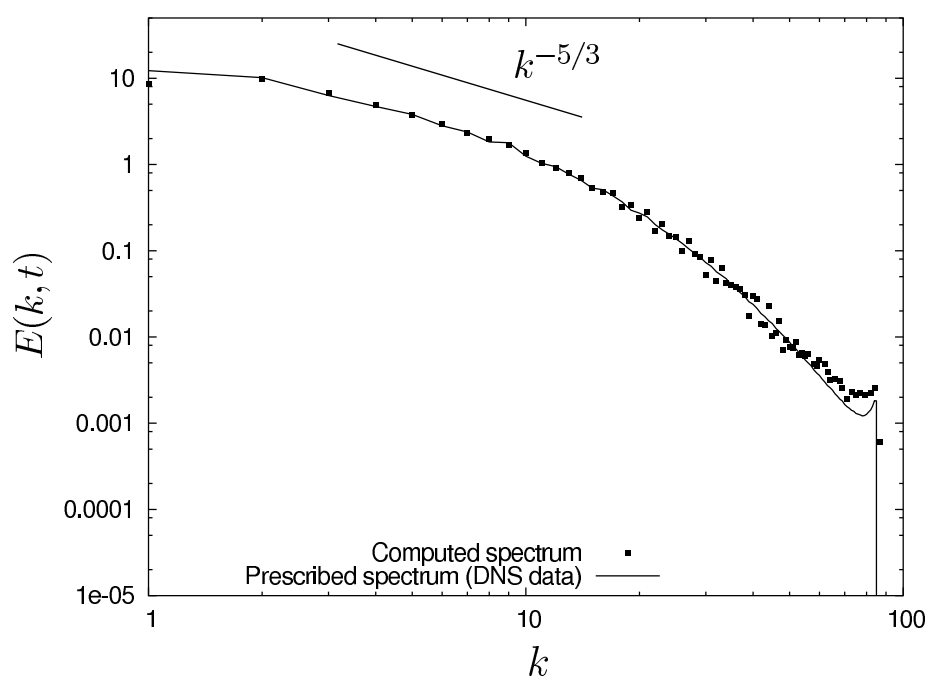

Figure 3: Energy spectrum in KS: comparison between the prescribed energy spectrum (obtained from forced DNS) and the calculated one with 2000 Fourier modes.

devoted to the inclusion of non-Gaussianity in the high-order spectra and related turbulence time-scales with experimental support [31], explicit data on the high-order spectra can be found.

\subsubsection{Unsteadiness in the isotropic case}

Since KS is mostly used in a Lagrangian framework, the unsteadiness of the synthetic flow is not generally studied in details. In many cases, the velocity field is frozen (i.e. $\omega_{n}=0$ for all $n$ in equation (4)) without any consequences on the results concerning one-particle dispersion (see for example figure 10 in [23]). When isotropic turbulence is considered, the unsteadiness is introduced via an arbitrary pseudo-dispersion law linking $\omega_{n}$ to the wave number $k_{n}$. Following [30], it can be chosen to be proportional to the eddy-turnover time of the $n^{\text {th }}$ wave mode

$$
\omega_{n}=\lambda \sqrt{k_{n}^{3} E\left(k_{n}\right)}
$$

where $\lambda$ is an arbitrary real parameter which controls the amount of unsteadiness of the flow. $\lambda$ is widely assumed to be around unity $[25,26,23,32]$. This choice of $\omega_{n}$ is often referred to as "local straining" and is a consequence of the local similarity approximation. It can be considered as a Lagrangian inverse timescale, since it depends on the local scale 
$1 / k_{n}$ and on its energy $E\left(k_{n}\right)$ only. The second possibility for defining $\omega_{n}$ comes from the sweeping hypothesis, in which an inverse Eulerian timescale is built as

$$
\omega_{n}=\lambda k_{n} u_{0}
$$

where $u_{0}$ is the rms velocity defined as

$$
u_{0}=\sqrt{\frac{2}{3} \int E(\kappa) \mathrm{d} \kappa} .
$$

These two hypotheses are used and compared in this paper.

Another distinction can be made concerning the nature of $\omega_{n}$, which can be defined as a deterministic or as a random variable. As introduced in [30], $\omega_{n}$ is considered in the following as a Gaussian random variable, with mean value $\lambda \varpi_{n}$ and standard deviation

$\lambda^{\prime} \varpi_{n}$, where $\varpi_{n}=\sqrt{k_{n}^{3} E\left(k_{n}\right)}$ in the straining hypothesis, and $\varpi_{n}=k_{n} u_{0}$ in the sweeping hypothesis. The choice of $\lambda^{\prime}=0$ corresponds to the classical case where the timescales are deterministic. The case $\lambda=\lambda^{\prime}=0$ is characteristic of a frozen velocity field.

\subsubsection{Unsteadiness in the rotating case}

We focus here on the linear dynamics of rotating turbulence, and its impact on the unsteadiness in KS models. The system of equations (1) is linearized and Fourier transformed. The solutions of this system in the Craya-Herring frame (see figure 2), are generated by a simple Green's function

$$
\left(\begin{array}{c}
u^{(1)}(\boldsymbol{k}, t) \\
u^{(2)}(\boldsymbol{k}, t)
\end{array}\right)=\left(\begin{array}{cc}
\cos \left[\sigma_{R}\left(t-t^{\prime}\right)\right] & -\sin \left[\sigma_{R}\left(t-t^{\prime}\right)\right] \\
\sin \left[\sigma_{R}\left(t-t^{\prime}\right)\right] & \cos \left[\sigma_{R}\left(t-t^{\prime}\right)\right]
\end{array}\right)\left(\begin{array}{c}
u^{(1)}\left(\boldsymbol{k}, t^{\prime}\right) \\
u^{(2)}\left(\boldsymbol{k}, t^{\prime}\right)
\end{array}\right)
$$

where $\sigma_{R}$ is the dispersion relation of inertial waves

$$
\sigma_{R}=2 \Omega \frac{\boldsymbol{k}}{k}
$$


Introducing the polar angle $\theta$ between the wave vector $\boldsymbol{k}$ and the rotation axis, equation (10) can be written $\sigma_{R}=2 \Omega \cos \theta$. Inertial waves are dispersive and the dispersion relation depends on the direction of the wave vector, but not on its modulus. As in $[29,20]$, the solutions (9) are explicitly included in equation (4), so that the Fourier

amplitudes $u^{(1)}$ and $u^{(2)}$ are now time-dependent. Given initial conditions derived from the prescribed energy spectrum, one can compute the velocity field at any subsequent time.

At this stage, the unsteadiness of the synthetic velocity field can be derived from two different processes: the classical arbitrary pulsation $\omega_{n}$ in isotropic cases and the dispersion relation of inertial waves in rotating cases. It is important to note that the two sources of unsteadiness are very different since the arbitrary pulsation $\omega_{n}$ affects all the Fourier modes, whereas the influence of the linear dynamics is different for the poloidal component $u^{(2)}(\boldsymbol{k}, t)$ and the toroidal component $u^{(1)}(\boldsymbol{k}, t)$, much as a phasemixing phenomenon.

\section{Two-time correlations of isotropic turbulence}

In this section, we focus on two-time Eulerian correlations of isotropic homogeneous steady turbulence, and we compare results obtained via DNS and KS. In both cases, we evaluate the two-time correlation of given velocity Fourier modes

$$
R(k, \tau)=\frac{<\hat{u}_{i}(\boldsymbol{k}, t) \hat{u}_{i}^{*}(\boldsymbol{k}, t+\tau)>}{<\hat{u}_{i}(\boldsymbol{k}, t) \hat{u}_{i}^{*}(\boldsymbol{k}, t)>} .
$$

The brackets $<\ldots>$ denote the average over time, realizations and direction of the wave vector $\boldsymbol{k}$. The normalization in (11) is relevant here since the energy spectra of the two numerical methods are the same. Thus, the two-time correlations of velocity Fourier modes are also the same for $\tau=0$. Following [10], we also introduce the correlation time 

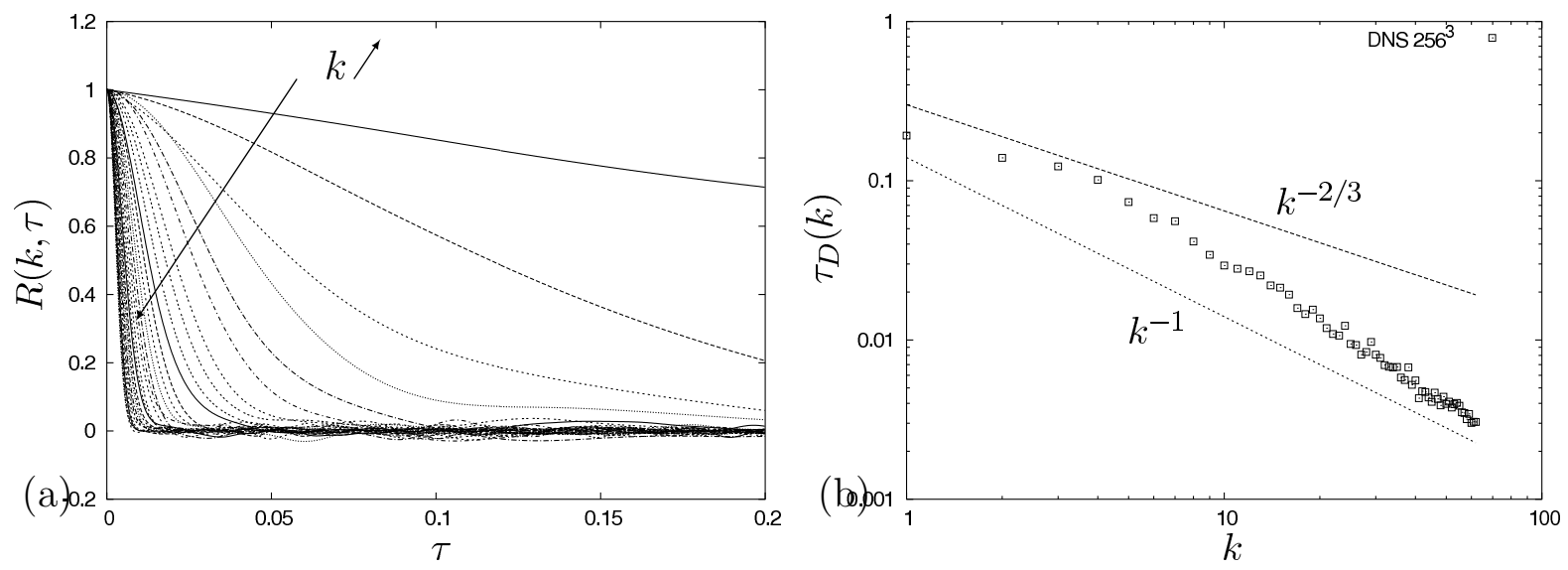

Figure 4: Two-time correlations in isotropic DNS for run DNS 1 in table 1. (a) Correlation function $R(k, \tau)$ as a function of $\tau$ for $k \in[2: 64]$. (b) Correlation time $\tau_{D}$ as a function of the wave number $k \in[2: 64]$.

$\tau_{D}(k)$ defined at a given scale $k$ by

$$
\tau_{D}(k)=\int_{0}^{\infty} R(k, \tau) \mathrm{d} \tau
$$

This definition of $\tau_{D}$ is not unique, one could for instance also choose the half-width of the correlation function.

\subsection{DNS results}

We first use equation (11) to compute $R(k, \tau)$ using DNS of isotropic turbulence. The statistics are averaged over three realizations of the flow obtained using different initial superposition of random Fourier modes. The parameters of the simulations presented in this section are gathered in table 1 . In figure $4(\mathrm{a})$, the coefficient $R(k, \tau)$ is plotted for wave numbers from $k=2$ to $k=64$. As the wave number increases, the correlation time decreases.

Under Kolmogorov's assumptions, the eddy-turnover time $\tau_{e}(k) \approx\left(k^{3} E(k)\right)^{-1 / 2}$ can be written as $\tau_{e}(k) \approx\left(\epsilon^{1 / 3} k^{2 / 3}\right)^{-1}$. The sweeping time is simply $\tau_{s}(k) \approx\left(u_{0} k\right)^{-1}$. These scaling laws are plotted in figure $4(\mathrm{~b})$ along with the correlation time $\tau_{D}(k)$ computed using DNS. As the wave number increases, the computed values of the correlation time 

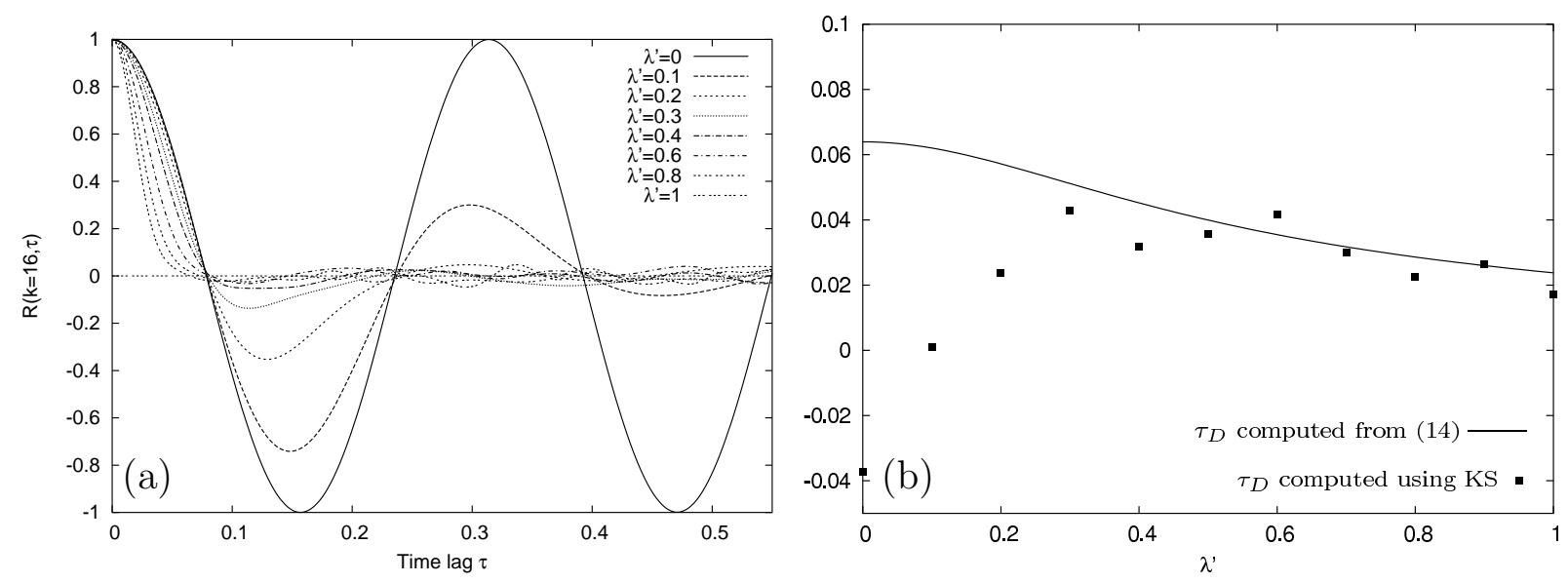

Figure 5: (a) Correlation function $R(k=16, \tau)$ for different values of $\lambda^{\prime}$. (b) Correlation time $\tau_{D}$ as a function of $\lambda^{\prime}$. The line corresponds to equation (14) whereas black squares are KS results (run KS 1 in table 1).

$\tau_{D}$ are much closer to the -1 slope than to the $-2 / 3$ slope. This confirms that the sweeping timescale is dominant at high wave numbers, thus at small scales. However, the conclusions remain unclear concerning the characteristic time of low wave numbers. These conclusions are similar to the ones obtained by $[10,12,33]$.

\subsection{KS results}

As presented in section 2.2.1, one advantage of KS is that the unsteadiness of the flow is explicitly controlled via the arbitrary choice of a correlation frequency $\omega_{n}$. We investigate the two main possibilities in the isotropic case, namely the straining hypothesis (6) and the sweeping hypothesis (7). Since $\omega_{n}$ is considered as a Gaussian variable, one can pilot its mean and average by choosing specific values for the parameters $\lambda$ and $\lambda^{\prime}$. The input and parameters of KS are therefore the prescribed spectrum $E(k)$ and the pair of variables $\lambda$ and $\lambda^{\prime}$. In most studies, $\lambda^{\prime}=0$, except [30] in which $\lambda^{\prime}=\lambda$ (some papers propose another way to randomize $\omega_{n}$, see for example [23]). $\lambda$ is often assumed to be close to unity, and a value around 0.5 is often encountered. The values of $\lambda$ and $\lambda^{\prime}$ investigated in the present paper can be found in table 1.

From equation (4), two-time correlations are analytically known in KS. The only source of unsteadiness in this case of isotropic turbulence is the Gaussian variable $\omega_{n}$ so 
that

$$
R\left(k_{n}, \tau\right)=<e^{-j \omega_{n} \tau}>=\exp \left(-\frac{1}{2} \omega_{n 0}^{2} \tau^{2}\right)
$$

where $\omega_{n 0}=\sqrt{\lambda^{2}+\lambda^{\prime 2}} \varpi_{n}$ is the $r m s$ value of $\omega_{n}$. The correlation time $\tau_{D}$ is then deduced as

$$
\tau_{D}=\frac{\sqrt{2 \pi}}{2 \omega_{n 0}} .
$$

In this case, time correlations depend only on the choice of $\lambda$ and $\lambda^{\prime}$, and on the relation $\varpi_{n}$. Considering the influence of the standard deviation on the correlation $R(k, \tau)$, we set the value of $\lambda$ at an arbitrarily fixed 0.4 and we focus on the impact of $\lambda^{\prime}$. The arbitrary law $\varpi\left(k_{n}\right)$ is here without importance and the unsteadiness of the velocity field is then derived from the straining hypothesis. Figure 5(a) presents the time dependent coefficient $R(k=16, \tau)$ for $\lambda^{\prime}$ increasing from 0 to 1 . The following results concerning the impact of $\lambda^{\prime}$ are independent of the wave number, and the specific choice of $k=16$ is made without loss of generality.

When $\lambda^{\prime}=0$, each Fourier mode at a given $k$ oscillates with the same frequency, defined by the pseudo-dispersion relation $\omega_{n}\left(k_{n}\right)$ (equations $(6)$ or $(7)$ ). Hence, at a given wave number, no decorrelation is observed, even for long time lag $\tau$. These oscillations are not observed in DNS in which negative loops of $R(k, \tau)$ are rare events (as in figure 4(a)). Note in addition that these oscillations vanish after integration over all wave numbers in equation (11), so that the dependence on the wave number in (11) is very important. When $\lambda^{\prime} \neq 0$, the standard deviation of $\omega_{n}$ is different from zero, the oscillations are strongly damped, and the correlation $R(k, \tau)$ rapidly goes to zero for large time lag. This is due to the fact that, at a given wave number, two different Fourier components can have different frequencies. This observation supports the use of a Gaussian variable instead of the classical deterministic law $\omega_{n}\left(k_{n}\right)$.

In figure $5(\mathrm{~b})$ the same results are presented after integration over time to yield $\tau_{D}$ as a function of $\lambda^{\prime}$. For not too small $\lambda^{\prime}$, the computed values of $\tau_{D}$ agree with the analytical result of equation (14), with $\omega_{n 0}=\sqrt{\lambda^{2}+\lambda^{\prime 2}} \sqrt{k^{3} E(k)}, k=16$ and $\lambda=0.4$. The 

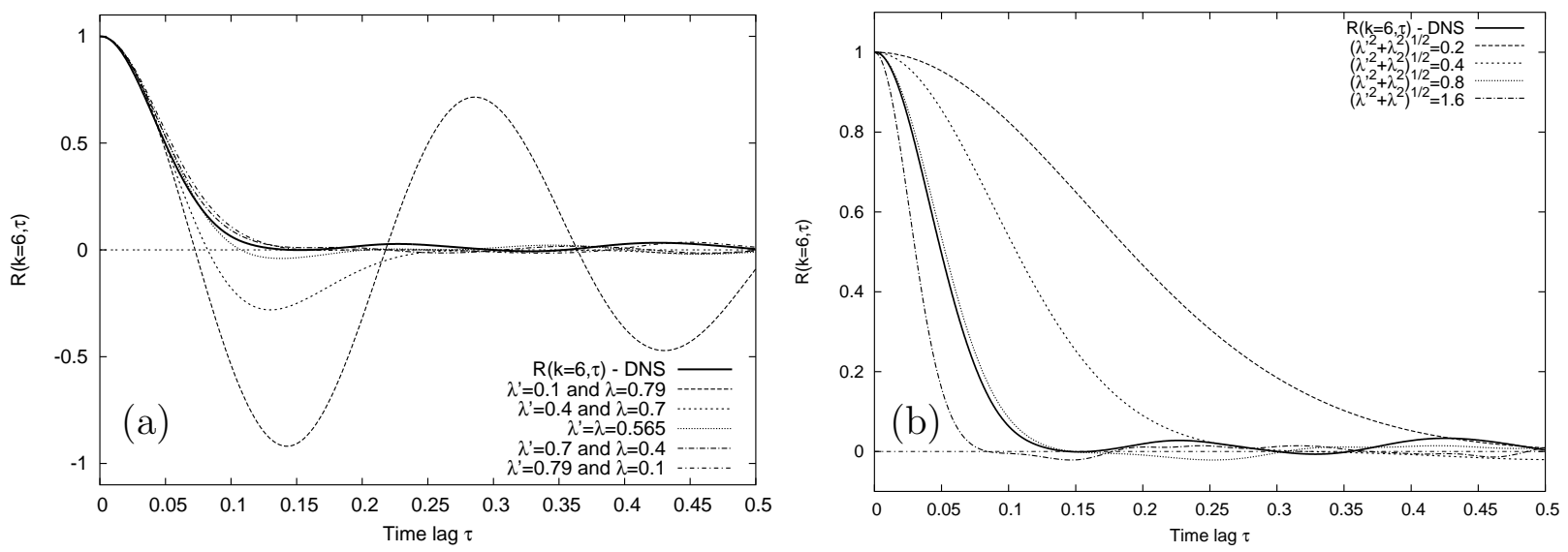

Figure 6: Correlations $R(k=6, \tau)$ obtained by DNS (continuous line) and KS (dashed lines) for different values of $\lambda$ and $\lambda^{\prime}$ (Run KS 2 in table 1). (a) The value of $\sqrt{\lambda^{2}+\lambda^{\prime 2}}$ is arbitrarily fixed to 0.8 and five different couples of variables are presented. (b) The value of $\sqrt{\lambda^{2}+\lambda^{\prime 2}}$ varies from 0.2 to 1.6 and the constraint $\lambda^{\prime}>\lambda$ is verified in each case.

disagreement for low values of $\lambda^{\prime}$ is due to the negative loops existing in the correlation function. All these qualitative results are unchanged for other values of $\lambda$ and $k$, as well as using the sweeping hypothesis. Note that in order to be sure that the negative loops of $R(k, \tau)$ vanish, $\lambda^{\prime}$ has to be larger than $\lambda$.

\subsection{Comparison between KS and DNS}

\subsubsection{Choice of $\lambda$ and $\lambda^{\prime}$}

In this section, we discuss how to specify values for $\lambda$ and $\lambda^{\prime}$. The following results depend on the parameters of the flow such as the Reynolds number. Given that $\lambda$ and $\lambda^{\prime}$ do not depend on the wave number, we present in the following a method to specify these quantities at a given wave number. The KS model uses the straining hypothesis but the comparison would have been exactly the same using the sweeping hypothesis.

Figure 6(a) presents the coefficient $R(k=6, \tau)$ computed using DNS and KS, for a given value of $\sqrt{\lambda^{2}+\lambda^{\prime 2}}=0.8$. The wave number $k=6$ corresponds to large energetic scales in the turbulent flow (see figure 1). The KS results are presented for five different values of $\lambda$ and $\lambda^{\prime}$ such that $\sqrt{\lambda^{2}+\lambda^{\prime 2}}=0.8$. As already observed, the negative loops 
in the correlation function $R(k=6, \tau)$ disappear when the constraint $\lambda^{\prime}>\lambda$ is satisfied. Note in addition that, for a given value of $\sqrt{\lambda^{2}+\lambda^{\prime 2}}$, the half-width of the correlation function is roughly independent of the particular values of $\lambda$ and $\lambda^{\prime}$. The KS results gathered in figure 6(b) correspond to different value of $\sqrt{\lambda^{2}+\lambda^{\prime 2}}$, and the constraint $\lambda^{\prime}>\lambda$ is satisfied in each case. The correlation function $R(k=6, \tau)$ obtained by DNS is well fitted by $f(\tau)=\exp \left(-275 \tau^{2}\right)$ (neglecting the small oscillations observed for $\tau>0.2)$. It is then possible to define a value of $\lambda$ and $\lambda^{\prime}$ such that DNS and KS correlations collapse since

$$
\begin{aligned}
R_{\mathrm{DNS}}(k=6, \tau) & \approx \exp \left(-275 \tau^{2}\right) \text { and } \\
R_{\mathrm{KS}}(k=6, \tau) & \approx \exp \left(-\frac{1}{2} \sqrt{\lambda^{2}+\lambda^{\prime 2}} k^{3} E(k) \tau^{2}\right)
\end{aligned}
$$

Looking at figure $3, E(k=6)=3.2$, so that equation (15) implies that $\sqrt{\lambda^{2}+\lambda^{\prime 2}} \approx 0.8$. Given the additional constraint $\lambda^{\prime}>\lambda$, it is then possible to derive different values for the couple of variables $\lambda$ and $\lambda^{\prime}$, at this given scale. Of course, the solution of the problem is not unique. In the following, we choose $\lambda=0.4$ and $\lambda^{\prime}=0.7$.

Once $\lambda$ and $\lambda^{\prime}$ are set, the time correlations of KS and DNS may be compared over all scales in the inertial range. The question is to find out which hypothesis, among the straining or sweeping ones, is the most relevant so that the correlation times are similar between KS and DNS at all scales.

\subsubsection{Comparison between sweeping and eddy-turnover times}

For a given range of wave numbers, a comparison of $\tau_{D}$ obtained with DNS and KS is proposed. The resolution in wave-number space is limited due to DNS although KS can be used to compute statistics with a broader energy spectrum than the one presented in figure 3. In section 3.2, we have seen that specific values for $\sqrt{\lambda^{2}+\lambda^{\prime 2}}$ can be found so that KS provides good correlations for a given wave number. We have chosen $k=6$ in section 3.3 in order to put the emphasis on the beginning of the inertial range, i.e. the 


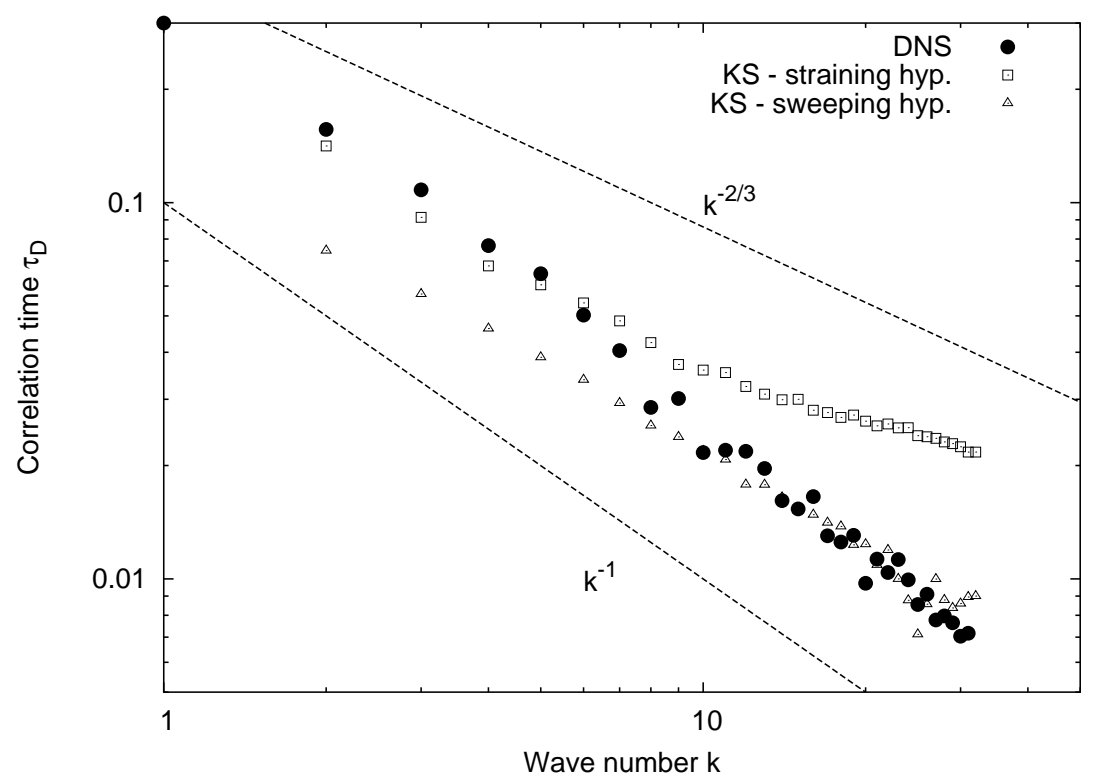

Figure 7: Comparison between correlation times in DNS and KS depending of the wave number: empty squares represent KS with eddy-turnover timescale, whereas empty triangles represent KS with sweeping timescale (Run DNS 1 and Runs KS 3 and 4 in table 1).

most energetic scales of the inertial range. Thus, in the following, we retain $\lambda=0.4$ and $\lambda^{\prime}=0.7$

A comparison of the correlation time $\tau_{D}$ obtained by DNS and $\mathrm{KS}$ is presented in figure 7. The eddy-turnover timescale is used to obtain the results represented by squares. The correlation times in DNS and KS agree in the low wave number range, as expected since $\lambda$ and $\lambda^{\prime}$ have been defined to that end in section 3.3. However, a difference appears as the wave number increases, showing that the KS eddies remain coherent for a longer time than the DNS ones. This version of the KS model with a Lagrangian point of view, in which the decorrelation of a given scale depends only on the size of this scale and on its energy, cannot predict the rapid decorrelation of the small scales of the flow. This departure from DNS results cannot be corrected using others values for $\lambda$ and $\lambda^{\prime}$ and the straining hypothesis alone is responsible for this departure.

Results of the KS model in which the sweeping timescale is used are represented by triangles in figure 7 . One observes that DNS and KS correlation times collapse at high wave numbers. The disagreement at low wave numbers can be attributed to many 
sources: the sweeping hypothesis is no longer valid for low wave numbers since the length scale is close to the one of the sweeping motion; the forcing of the DNS may enhance the correlation of forced wave numbers; and, finally, pseudo-spectral codes are known for their lack of accurate statistics at low wave numbers, due to discretization.

It is remarkable that there is no need to change the values of $\lambda$ and $\lambda^{\prime}$ obtained in section 3.3 when the law $\varpi_{n}$ is switched from straining to sweeping timescale. This suggests that the straining hypothesis is valid for low wave numbers whereas the sweeping hypothesis becomes dominant as the wave number increases.

\section{Two-time correlations of rotating turbulence}

The aim of this section is to characterize the effect of rotation on the statistical quantities previously studied in an isotropic context. We are interested in two-time Eulerian velocity correlations of rotating homogeneous quasi-steady turbulence. This section is devoted to the comparison between results obtained via DNS and KS including the explicit linear dynamics of inertial waves (see section 2.2.2). The rotation axis is assumed to be vertical in the following. In an axisymmetric configuration, the correlation function (11) can be written in a more general form

$$
R(k, \theta, \tau)=\frac{<\hat{u}_{i}(k, \theta, t) \hat{u}_{i}^{*}(k, \theta, t+\tau)>}{<\hat{u}_{i}(k, \theta, t) \hat{u}_{i}^{*}(k, \theta, t)>}
$$

where $\theta$ is the polar angle between the rotation axis and the direction of the wave vector. This time, the brackets $\langle\ldots\rangle$ denote the average over time, realizations and the azimuthal angle. Since the inertial waves are dispersive and the dispersion relation (10) is anisotropic, it is important to retain the dependence on the wave number $k$ and on $\theta$ in (16). 

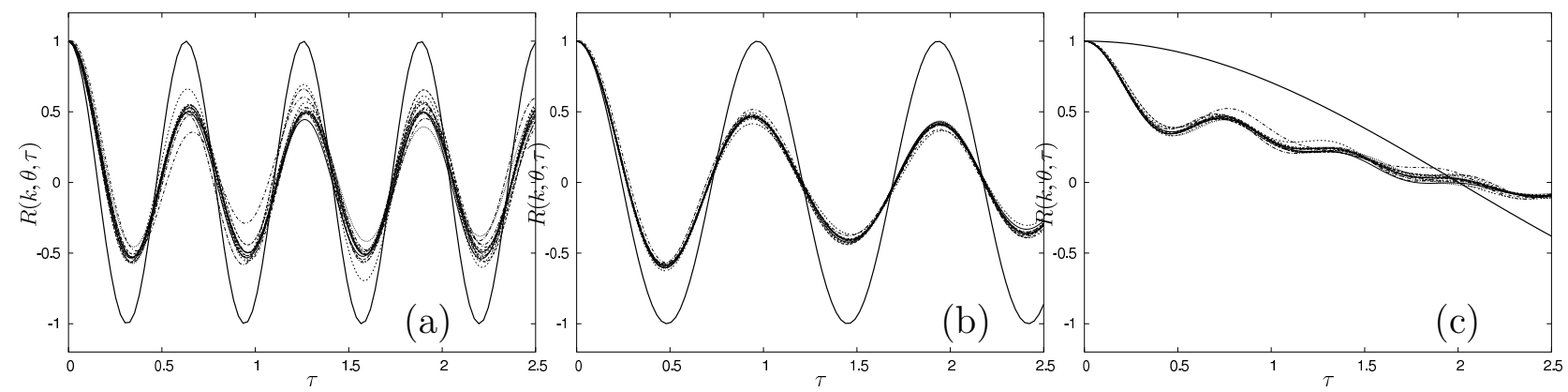

Figure 8: Two-time correlation functions $R(k, \theta, \tau)$ for $k \in[2: 64]$ computed using KS including linear dynamics and for different polar angles. Each light curve corresponds to a different $k$. The thick line represents $\cos (2 \Omega \cos (\theta) \tau)$, with $\Omega=10 \mathrm{rad}_{\text {. }}{ }^{-1}$. (a) $\theta \approx 0$. (b) $\theta \approx \pi / 4$. (c) $\theta \approx \pi / 2$. (Run KS 5 in Tab.1).

\subsection{KS results in the purely rotating case}

In a first approach, we investigate the effect of solid-body rotation on two-time correlations in the linear limit. The constants $\lambda$ and $\lambda^{\prime}$ are set to zero so that the inertial waves are alone responsible for the unsteadiness of the synthetic flow. The rotation rate is $\Omega=10 \mathrm{rad} . \mathrm{s}^{-1}$. The advantage of $\mathrm{KS}$ in this context is to separate the effect of the linear dynamics from the decorrelation existing in isotropic turbulence (previously simulated by the straining and the sweeping hypotheses). Figure 8 presents the correlation defined in (16) for three different angular domains. The first one corresponds to $\theta \approx 0$ (vertical modes aligned with the rotation axis), the second one to $\theta \approx \pi / 4$ and the third one to $\theta \approx \pi / 2$ (horizontal modes perpendicular with the rotation axis). Given that the pulsation of inertial waves depends only on the direction of the wave vector, one expects that all the modes oscillate with the same frequency for a given value of $\theta$. This behavior is observed in figure $8(\mathrm{a})$, where the function $R(k, \theta, \tau)$ is plotted for $\theta \approx 0$ and $2<k<64$. The thick line represents the function $\cos (2 \Omega \tau)$ which is the theoretical correlation function for vertical modes. As the polar angle $\theta$ increases, the pulsation of inertial waves decreases. In the limit $\theta \rightarrow \pi / 2$, the Fourier modes are almost unaffected by rotation and tend to a stationary state (see figure $8(\mathrm{c})$ ). 

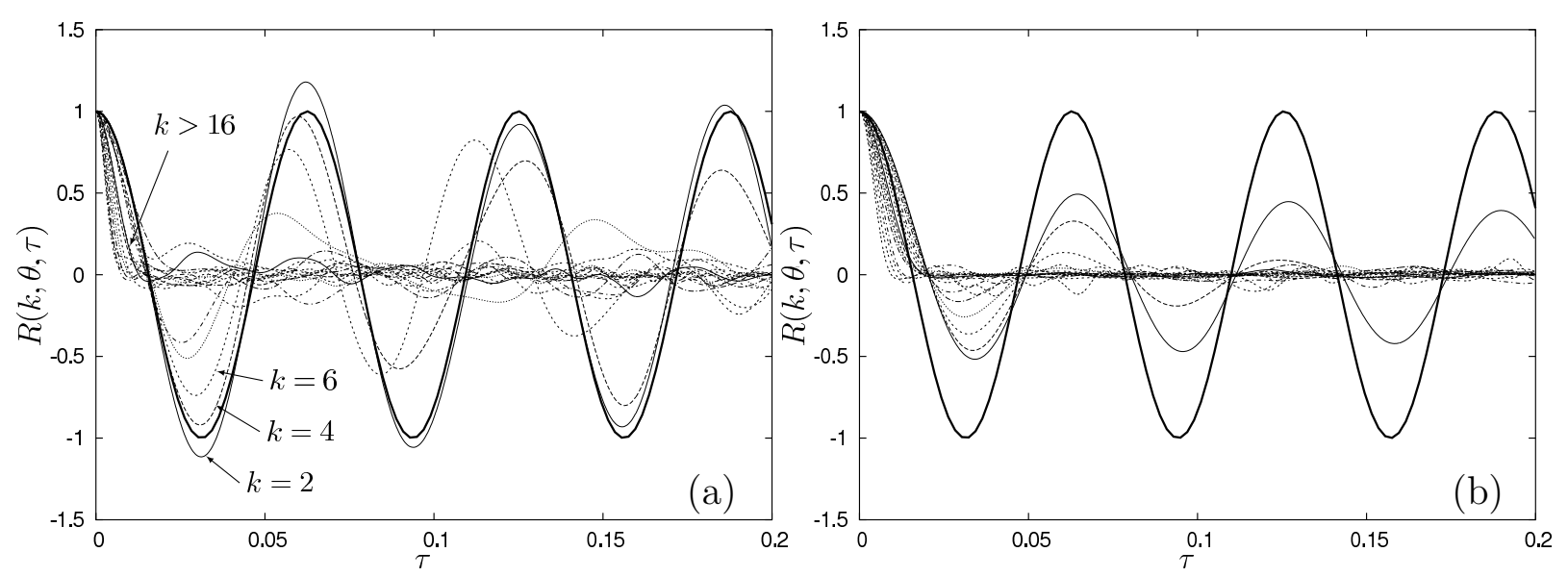

Figure 9: Two-time correlation function $R(k, \theta, \tau)$ for $k \in[2: 64]$ and $\theta \approx 0$. The Rossby number is approximately 0.05 . The thick line corresponds to the function $\cos (2 \Omega \tau)$. (a) DNS. (b) KS. (Runs DNS 2 and KS 6 in table 1).

\subsection{Comparison between DNS and KS}

In this section, the correlation function (16) is computed using the pseudo-spectral DNS method presented in section 2.1. The reference frame is rotating with a rotation rate $\Omega=50 \mathrm{rad} . \mathrm{s}^{-1}$, corresponding to a Rossby number about 0.05 . We computed three independent realizations of the flow so that the refined angular statistics are reasonably converged. It is of course possible to compare the DNS results with KS including both linear dynamics due to rotation and arbitrary pulsation. The values for $\lambda$ and $\lambda^{\prime}$ are the same as in section 3.3 and the rotation rate is the same as in DNS, i.e. $\Omega=50 \mathrm{rad} . \mathrm{s}^{-1}$.

Figure 9 (to be compared with figure 4(a)) presents the correlation function of nearly vertical modes (i.e. such that $\theta \approx 0$ ) computed using DNS and KS. In the linear limit, the case $\theta \approx 0$ corresponds to inertial waves with the maximum pulsation $2 \Omega$. Despite the relatively coarse spectral resolution, one can separate two main domains: low wave numbers influenced by inertial waves and high wave numbers very similar to the nonrotating case.

The half-width time $\tau_{1 / 2}$ is a better quantity than the integral time $\tau_{D}(12)$ to char- 
acterize the effect of rotation and is defined from

$$
R\left(k, \tau_{1 / 2}\right)=\frac{1}{2}
$$

Figure 10 presents $\tau_{1 / 2}(k)$ computed using DNS and KS for discrete values of the polar angle $\theta$. Horizontal modes (represented by $\mathbf{\Delta}$ ) are almost unchanged by rotation and the sweeping timescale is dominant, especially at high wave numbers. Modes such that $\boldsymbol{\Omega} \cdot \boldsymbol{k} \neq 0$ are influenced by rotation only at low wave numbers, for which the half-width time is roughly independent of the wave number. This is consistent with the preceeding linear analysis, predicting a correlation time that depends on the direction of the wave vector only. The transition between the two regimes can be found by equating the pulsation of inertial waves and the pulsation of the sweeping effect leading to

$$
2 \Omega \cos \theta \approx u_{0} k_{c}
$$

For vertical modes, the pulsation of inertial waves is maximum so that $k_{c}$ is maximum and the rotation influences a large range of wave numbers. As the polar angle $\theta$ increases, the pulsation of inertial waves decreases and the effect of rotation is dominant only at low wave numbers. The results presented in figure 10 are obtained with $\Omega=50 \mathrm{rad} . \mathrm{s}^{-1}$ ( $R o \approx 0.05)$ and $u_{0} \approx 6$, so that the critical wave number for vertical modes is $k_{c} \approx 16$, in good agreement with the transition observed in figure 10(a). This analysis enhances that the effect of background rotation depends on the wave number and on the direction of propagation.

Here lies a significant difference between rotating turbulence and sheared turbulence. On the one hand, the breaking of turbulence isotropy by rotation is subtle. The linear solution has no direct impact on single-time second-order statistics of initially isotropic turbulence, so that the energy spectrum is not affected (the time dependence induced by the phase term in the linear operator cancels out by multiplying $e^{\mathrm{i} \sigma_{R} t}$ by its complex conjugate, see the linear solutions of the system (9)). However, when considering two- 

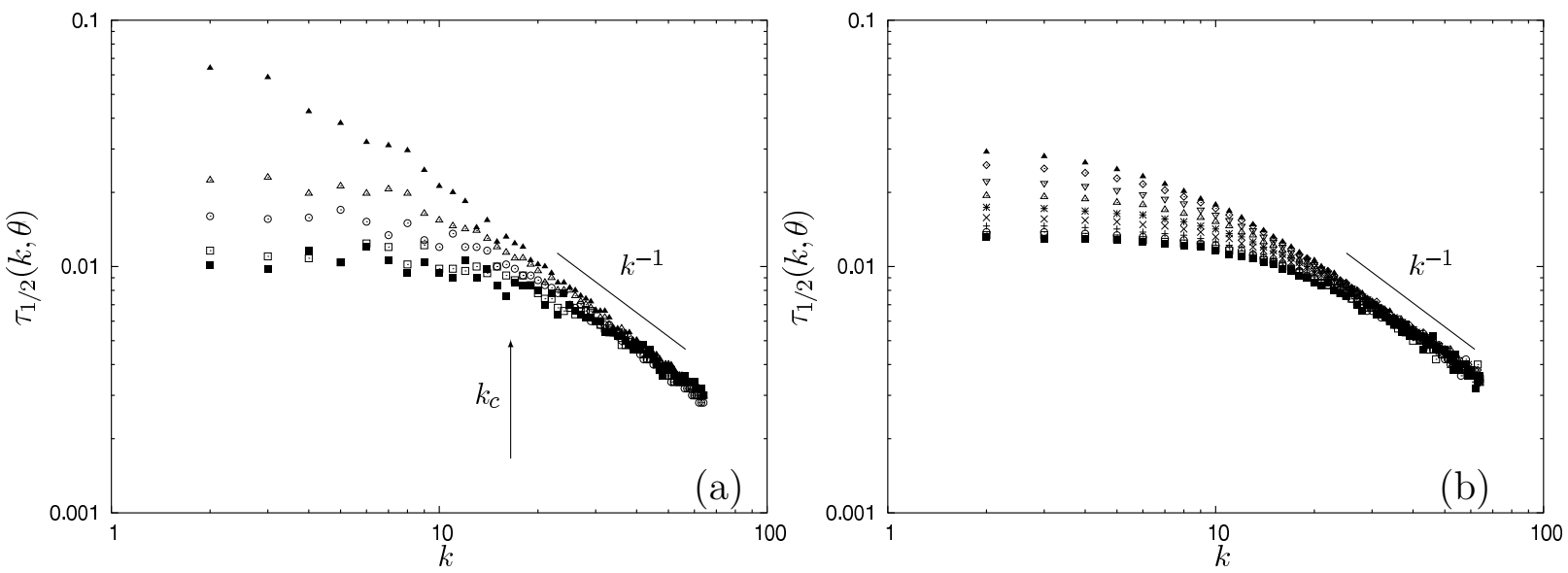

Figure 10: Correlation times $\tau_{1 / 2}(k, \theta)$ for $k \in[2: 64]$ and discrete values of $\theta$ between $\boldsymbol{\Delta} \theta \approx \pi / 2$ and $\boldsymbol{\square} \theta 0$. (a) DNS. (b) KS.

time second order statistics, isotropy is broken by this linear operator (because of nontrivial phase-mixing induced by $e^{\mathrm{i} \sigma_{R}\left(t-t^{\prime}\right)}$ if $t \neq t^{\prime}$; see [15] for details). On the other hand, the energy spectrum is directly affected by the mean shear, so that it could be no longer considered as constant, in the presence of a mean shear flow [16]. In addition, the linear RDT solution involves a time-dependent wavevector in sheared turbulence, which reflects linear advection and cannot be removed by a change of frame as in the rotating case.

\section{An application to aeroacoustics}

The frequency of the sound radiated by turbulent flows is related to the temporal decorrelation of the turbulent scales. The costs of DNS increase dramatically in the aeroacoustic framework since the whole temporal evolution of the flow has to be stored. One solution involves a method combining LES for computing the turbulent sources and an acoustic analogy (or the linearized Euler equations). However, this suggests to study the effect of the subgrid scale model (SGS) on time correlations of turbulent flows and to understand the impact of the unresolved scales of the flow on acoustic radiation $[34,35,12]$.

In that context, $\mathrm{KS}$ is a good candidate to compute the small scale velocity field, unresolved by LES. This approach has already been used to study properties of scalar 
variances in turbulent flows [36] and particle deposition in a plane channel with a ribbed wall [32]. KS has also been used to compute interaction terms between the mean flow and turbulent fluctuations in the linearized Euler equations [28]. An application of KS used in addition of LES in an aeroacoustic context can be found in [37]. Note that, in addition to KS, a SGS model is still needed to solve the large scale motion of the flow. This aspect is beyond the scope of this paper (see [36] for more details). Considering the results of the preceding sections, time correlations of KS are closely linked to the choice of $\omega_{n}$. Time decorrelations of small scales have to be modeled by a Gaussian characteristic frequency (to avoid oscillations of $R(k, \tau)$ ) and a timescale based on the sweeping hypothesis has to be used. These restrictions could have a considerable impact on the subgrid-scale velocity field computed by KS, especially in an aeroacoustics framework.

Moreover, given the good agreement between rotating DNS and KS including linear solutions, it is interesting to compare the acoustic emission predicted by these two models, in the case of rotating turbulence. The following part is devoted to the computation of the sound emitted by isotropic and rotating turbulence. Both DNS and KS including linear dynamics are used and Lighthill's analogy is used to derive acoustic properties of the flow from hydrodynamic quantities.

\subsection{Lighthill's theory}

The sound emission of isotropic steady turbulence is computed using Lighthill's analogy [19]. By re-arranging the Navier-Stokes equations, Lighthill established the following relation

$$
p(\boldsymbol{x}, t)=\frac{1}{4 \pi c_{0}^{2}} \frac{x_{i} x_{l}}{|\boldsymbol{x}|^{3}} \int_{V} \frac{\partial^{2}}{\partial t^{2}} T_{i l}\left(\boldsymbol{y}, t-\frac{|\boldsymbol{x}-\boldsymbol{y}|}{c_{0}}\right) d^{3} \boldsymbol{y}
$$

where $p(\boldsymbol{x}, t)$ is the fluctuating pressure calculated at $\boldsymbol{x}, c_{0}$ the speed of sound, $V$ the volume of turbulent sources and $T_{i l}=\rho_{0} u_{i} u_{l}$ the Lighthill's tensor (the entropy noise and the viscous term are neglected). Equation (19) emphasizes the dependence of the sound pressure on the two-point (due to integration over $V$ ) and two-time (due to the 


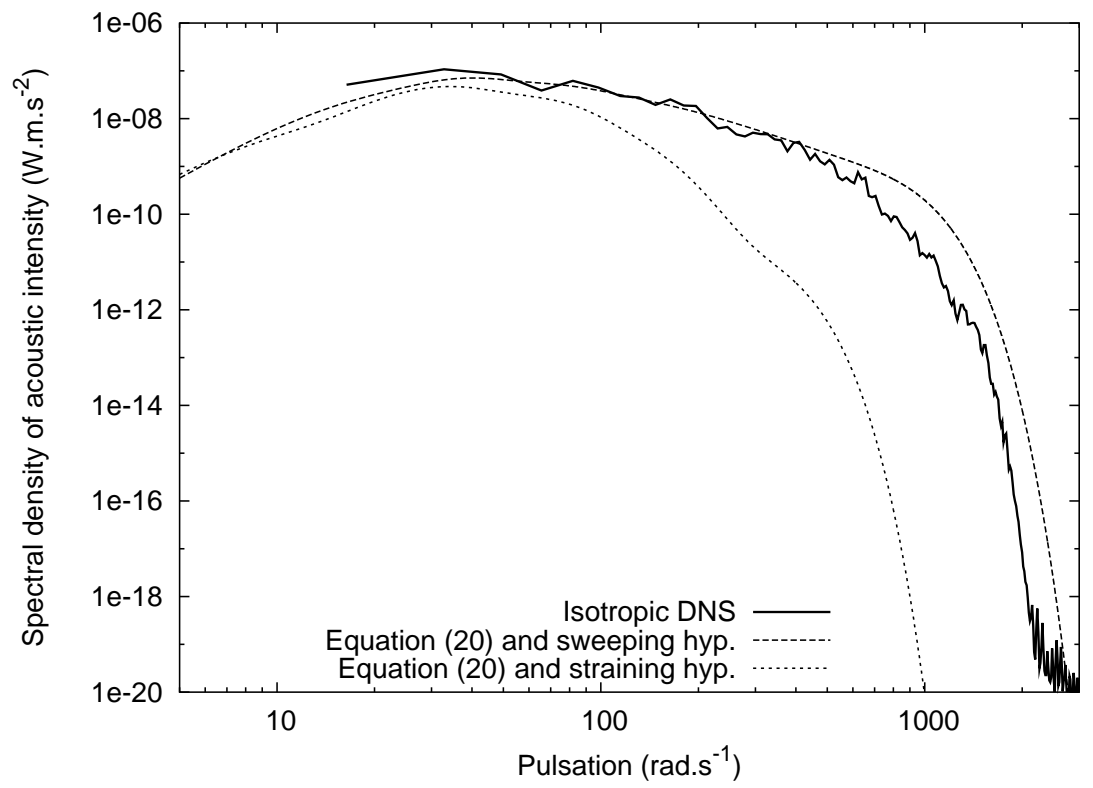

Figure 11: Comparison between acoustic power spectra obtained by isotropic DNS and equation (20). (Run DNS 1 in table 2).

retarded time $\left.t-|\boldsymbol{x}-\boldsymbol{y}| / c_{0}\right)$ velocity correlations. The use of this acoustic formulation is also supported by the validation of Lighthill's relationship on fourth order correlations by experimental measurements provided in [38].

Assuming the quasi-normality of the fourth-order velocity correlations and using the compact source assumption, it is thus possible to derive an analytical formulation for the sound emitted by isotropic turbulence $[13,14]$. The acoustic power spectral density function written in the form

$$
P_{T}(\omega)=\frac{\pi}{2} \rho_{0} \frac{\omega^{4}}{c_{0}^{5}} \frac{32 \pi}{15} \int_{0}^{\infty} 4 \pi k^{2} \frac{E^{2}(k)}{\left(4 \pi k^{2}\right)^{2}}\left(\frac{1}{2 \pi} \int_{-\infty}^{\infty} R^{2}(k, \tau) e^{-j \omega \tau} \mathrm{d} \tau\right) \mathrm{d} k
$$

depends only on the energy spectrum $E(k)$ and the time correlations function $R(k, \tau)$. This formulation is of particular interest here since these two functions are also the two inputs needed in $\mathrm{KS}$. 


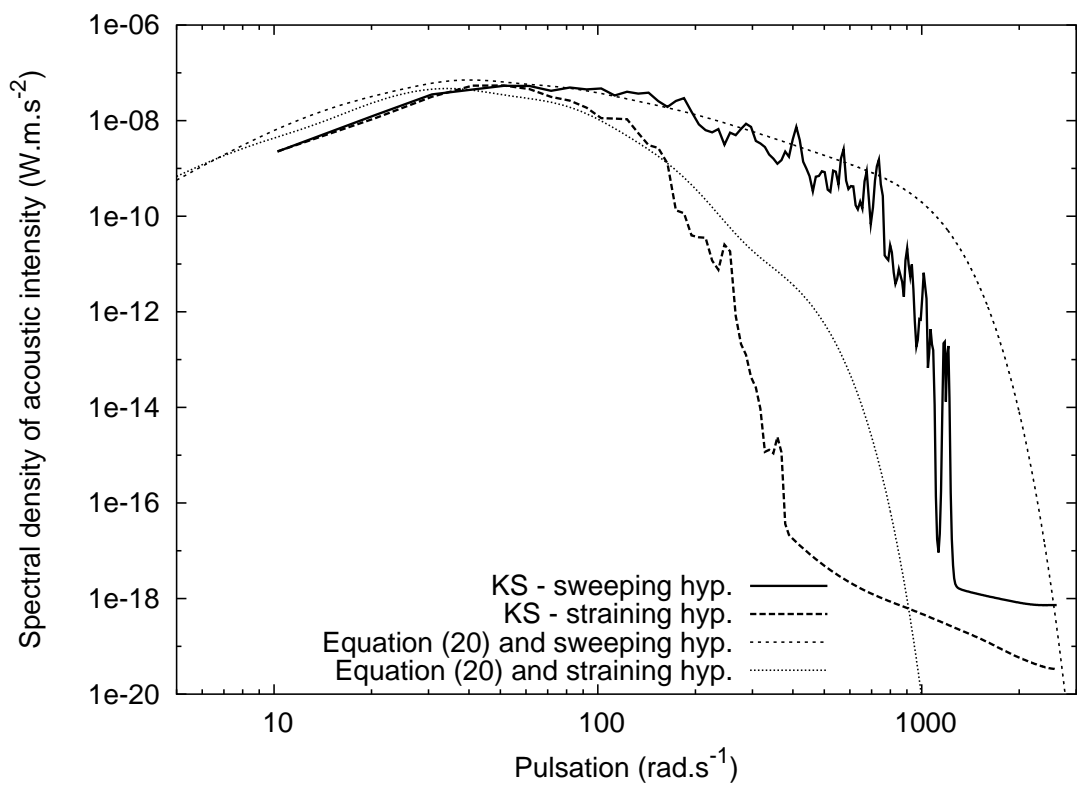

Figure 12: Comparison between acoustic power spectra obtained by isotropic KS and equation (20). (Runs KS 1 and 2 in table 2).

\subsection{Results for isotropic turbulence}

In this section, we compute the acoustic intensity of isotropic turbulence using three different approaches: (a) DNS, (b) KS and (c) equation (20). The numerical parameters of the simulations presented in this section are gathered in table 2. Both eddy-turnover and sweeping timescales are explored when it is possible, i.e. using $\mathrm{KS}$ and equation (20). On the one hand, equation (19) is used to compute the sound emitted by velocity fields derived from DNS and KS. The same algorithm is used in both cases. Details about the numerical aspect of the problem can be found in $[39,40,20]$. On the other hand, equation (20) is integrated directly using the same energy spectrum $E(k)$ as for DNS and KS (i.e. the quasi-steady energy spectrum of forced DNS) and the sweeping (i.e. $\left.R(k, \tau)=\exp \left(-k^{2} u_{0}^{2} \tau^{2} / 2\right)\right)$ or straining (i.e. $\left.R(k, \tau)=\exp \left(-k^{3} E(k) \tau^{2} / 2\right)\right)$ hypotheses.

Figure 11 presents the comparison between DNS and equation (20). Using the eddyturnover timescales in equation (20) leads to underestimating the sound emitted at high frequencies. It is clear that the sweeping timescale is much more efficient to estimate the sound emitted by the small scales of the flow simulated by DNS. The same com- 

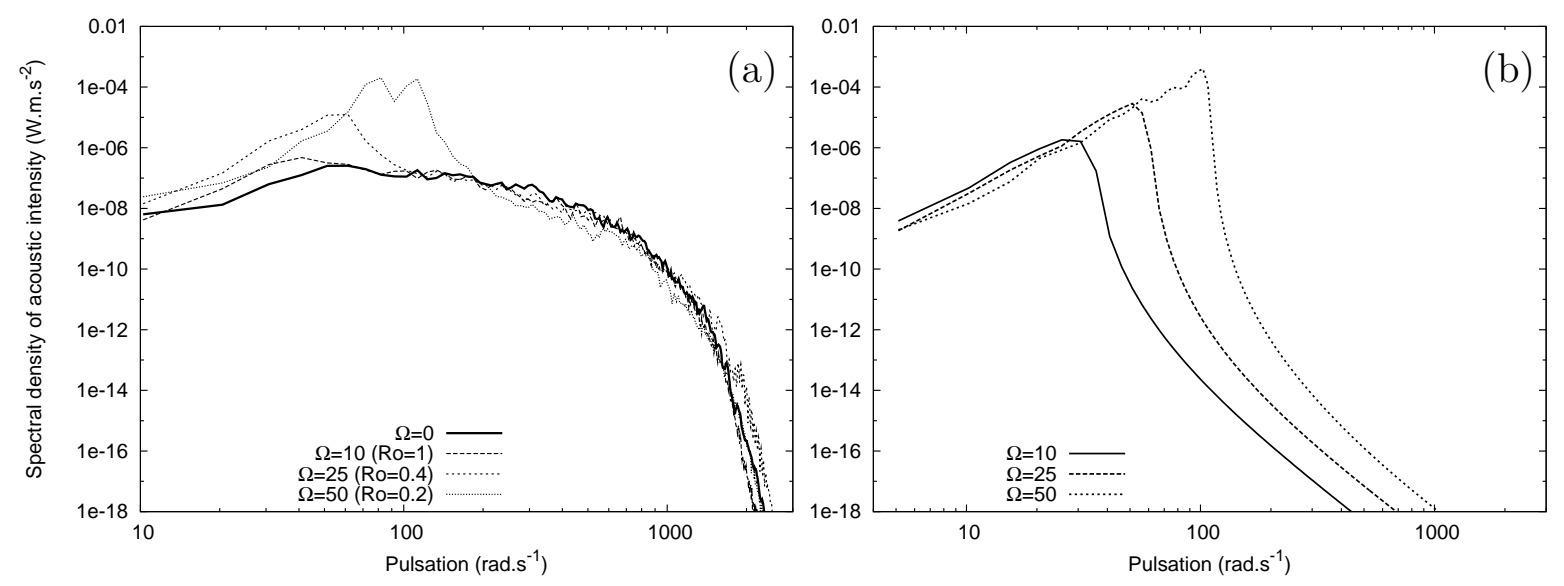

Figure 13: Acoustic spectra of homogeneous rotating turbulence. (a) DNS (run DNS 2 in table 2). (b) KS without arbitrary pulsations but including the linear solutions of eq.(9) (run KS 3 in table 2).

parison is presented in figure 12 , for $\mathrm{KS}$ results. These results illustrate the potential of $\mathrm{KS}$ as a diagnostic tool: it is possible to compare the influence of the two hypotheses discussed below in an unique model. The conclusions are the following: the acoustic power spectra computed from KS and equation (20) are very similar, especially at low frequencies. In addition, these results confirm the importance of the sweeping timescale in the acoustic emission by the small scales of a turbulent flow. KS therefore appears to be a possible model, with low numerical cost, to compute the small scale velocity field part in aeroacoustic studies, provided that the sweeping timescale is introduced [37].

We have also performed KS with different values of $\lambda$ and $\lambda^{\prime}$ (not reported here). The acoustic emission of the synthetic model is very sensitive to the value of $\sqrt{\lambda^{2}+\lambda^{2}}$ (i.e. to the half-width of the correlation function $R(k, \tau)$ ), but not to the particular values of $\lambda$ and $\lambda^{\prime}$ (i.e. the additional constraint $\lambda^{\prime}>\lambda$ observed in section 3.3 is not very important concerning the acoustic properties of $\mathrm{KS}$ ).

\subsection{Results for rotating turbulence}

In this section, we move on to the acoustic emission of rotating turbulence. Figure 13(a) shows the acoustic spectra computed using DNS and for different rotation rates. The Rossby number varies between unity and $10^{-1}$. As the Rossby number decreases, one 
can observe a dominant contribution from low-frequency components of the spectrum. The rotation does not seem to modify the acoustic emission at high frequency.

The main interest of KS is to allow the separate use of different sources of unsteadiness. First, we observe the acoustic emission of a superposition of inertial waves. The arbitrary pulsation $\omega_{n}$ is not considered in a first approach $\left(\lambda=\lambda^{\prime}=0\right)$ so that the unsteadiness of the synthetic field is based on linear solutions of (9) only, and depends on the dispersion relation of inertial waves. Figure 13(b) shows the acoustic spectra computed from this simple KS model, for different rotation rates and with $\lambda=\lambda^{\prime}=0$. These simulations correspond to the acoustic emission of rotating turbulence in the linear limit. A cut-off frequency is clearly observed, beyond which the rotation does not influence the acoustic emission. Moreover, this critical frequency is close to the maximum value of the pulsation of inertial waves $\omega_{\max }=2 \Omega$. For $\Omega=50 \mathrm{rad} . \mathrm{s}^{-1}, \omega_{\max } \approx 100 \mathrm{rad} . \mathrm{s}^{-1}$, which is consistent with the cut-off frequency observed in figures 13(b) and 13(a). Of course, DNS results display high frequency components due to the small-scales of the turbulent flow with characteristic times much smaller that of inertial waves.

As already mentioned in section 4.2 , it is possible to superimpose the two sources of unsteadiness in KS: namely the characteristic pulsation $\omega_{n}$ and the linear dynamics of rotating fluid. In addition, the random pulsation $\omega_{n}$ is reintroduced with $\lambda=0.4$ and $\lambda^{\prime}=0.7$, and is based either on the straining or on the sweeping hypotheses. Figure 14 presents the acoustic spectra computed using $\mathrm{KS}$ for $\Omega=25 \mathrm{rad} . \mathrm{s}^{-1}$ and compared to the equivalent DNS ones. The agreement of the KS spectra with the DNS one is very good, all the more when the sweeping hypothesis is used. It demonstrates that, when modeling the acoustic emission, both the following ingredients are necessary: on the one hand, the use of linear solutions is responsible for the enhanced low-frequency acoustic emission; on the other hand, the sweeping hypothesis is a good approximation to compute the small-scales decorrelation and the high frequencies acoustic emission. These conclusions, similar to the ones gathered in section 4.2 , demonstrate the close relation existing between two-time correlations and the acoustic emission of homogeneous turbulence. 


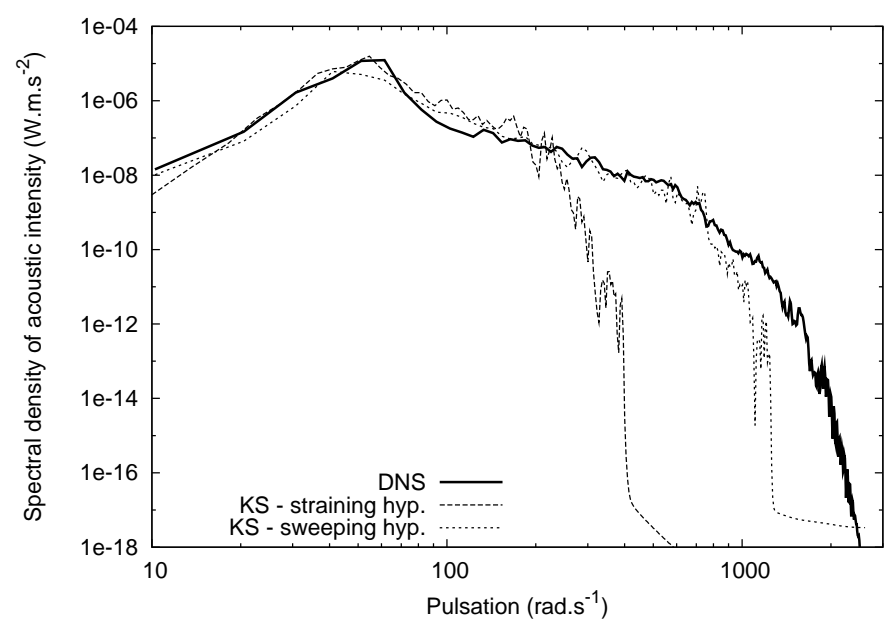

Figure 14: Acoustic spectra of homogeneous rotating turbulence. The rotation rate is $\Omega=25 \mathrm{rad} . \mathrm{s}^{-1}$. Comparison between DNS (run DNS 2 in table 2) and KS (runs KS 4 and 5 in table 2).

The sound radiated by rotating turbulence is directionally dependent, even in the far-field approximation. Additional acoustic results concerning the effect of rotation on the dependence of the sound emitted with the Mach number, or the directivity of the acoustic emission, can be found in [20].

\section{Conclusion}

In this paper, the time correlations of Fourier velocity modes are computed using DNS and KS. They are also related to two-point two-time correlations in physical space. Both isotropic and rotating homogeneous turbulence have been considered. The turbulence is quasi-steady, since DNS is forced at low wave numbers, and KS does not include the viscous decay. In the isotropic case, time correlations are similar in the two approaches provided two constraints are met in the KS model: the characteristic frequency $\omega_{n}$ has to be Gaussian (at least random) for each Fourier modes in equation 4, and the sweeping timescale $\omega_{n} \approx k_{n} u_{0}$ has to be used, at least for high values of $k_{n}$. In the rotating case, the linear dynamics, based on the dispersion relation of inertial waves existing in rotating flows, are explicitly included in KS. This simple approach allows to reproduce 
qualitatively the two-time correlations computed using DNS. The competition between the sweeping effect and the rotation is scale- and angle-dependent so that two spectral regions can be identified: the small wave numbers aligned with the rotation axis are dominated by rotation, whereas small scales or intermediate scales perpendicular to the rotation axis behave more similarly to the isotropic case and are dominated by the sweeping effect.

A direct application is the sound emission by turbulent flows, that strongly depends on two-time correlations. In this context, the acoustic emission of turbulent sources computed by KS has been studied, in which the unsteadiness is derived from both sweeping and eddy turnover assumptions. The frequency spectrum is compared with the one computed from a direct simulation of isotropic turbulent sources. Using the eddy turnover timescale in KS underestimates the acoustic power spectrum for high frequencies. An important improvement is found using a sweeping timescale, in which each Fourier modes are advected by the rms velocity of the flow. Concerning rotating flows, DNS and KS including linear dynamics have been performed. In the linear limit, the acoustic emission of rotating turbulence is altered by the presence of inertial waves. The linear KS results are very similar to rotating DNS results, with an enhancement of the acoustic emission at low frequencies, excited by inertial waves.

The authors would like to acknowledge the support of IDRIS (Institut du Développement et des Ressources en Informatique Scientifique) for computational time on NEC SX8, under project number 082206. 


\section{References}

[1] Y. Kaneda and T. Ishida, Suppression of vertical diffusion in strongly stratified turbulence, J. Fluid Mech. 402 (2000), pp. 311-327.

[2] I. Proudman, The generation of noise by isotropic turbulence, Proc. Roy. Soc. London Ser. A A 214 (1952), p. 119.

[3] G.M. Lilley, The radiated noise from isotropic turbulence revisited, Theor. Comput. Fluid Dyn. 6 (1994), p. 281.

[4] A.N. Kolmogorov, The local structure of turbulence in incompressible viscous fluid for very large Reynolds numbers, C.R. Acad. Sci. U.R.S.S. 36 (1941), p. 301.

[5] R.H. Kraichnan, Kolmogorov's hypotheses and Eulerian turbulence theory, Physics of Fluids 7 (1964), pp. 1723-1734.

[6] H. Tennekes, Eulerian and Lagrangian time microscales in isotropic turbulence, J. Fluid Mech. 67 (1975), pp. 561-567.

[7] S. Chen and R.H. Kraichnan, Sweeping decorrelation in isotropic turbulence, Physics of Fluids A 1 (12) (1989), pp. 2019-2024.

[8] G. Comte-Bellot and S. Corrsin, Simple Eulerian time correlations of full and narrow-band velocity signals in grid generated 'isotropic' turbulence, J. Fluid Mech. 48 (1971), p. 273.

[9] C. Poulain et al., Dynamics of spatial Fourier modes in turbulence, Eur. Phys. J. B 53 (2006), pp. 219-224.

[10] T. Sanada and V. Shanmugasundaram, Random sweeping effect in isotropic numerical turbulence, Physics of Fluids A 4 (6) (1992), pp. 1245-1250. 
[11] W.D. McComb, V. Shanmugasundaram, and P. Hutchinson, Velocity-derivative skewness and two-time velocity correlations of isotropic turbulence as predicted by the LET theory, J. Fluid Mech. 208 (1989), p. 91.

[12] G.W. He, M. Wang, and S.K. Lele, On the computation of space-time correlations by large-eddy simulation, Physics of Fluids 16 (2004), pp. 3859-3867.

[13] Y. Zhou and R. Rubinstein, Sweeping and straining effects in sound generation by high Reynolds number isotropic turbulence, Physics of Fluids 8 (3) (1995), pp. 647-649.

[14] R. Rubinstein and Y. Zhou, The frequency spectrum of sound radiated by isotropic turbulence, Physics Letters A 267 (2000), pp. 379-383.

[15] C. Cambon et al., Turbulent diffusion in rapidly rotating flows with and without stable stratification, J. Fluid Mech. 499 (2004), pp. 231-255.

[16] X. Zhao and G.W. He, Space-time correlations of fluctuating velocities in turbulent shear flows, Phys. Rev. E 79 (2009), p. 046316.

[17] L. Liechtenstein, F.S. Godeferd, and C. Cambon, Nonlinear formation of structures in rotating stratified turbulence, Journal of Turbulence 6 (2005).

[18] P. Sagaut and C. Cambon Homogeneous turbulence dynamics, Cambridge University Press, 2008.

[19] M. Lighthill, On sound generated aerodynamically, I General theory, Proc. Roy. Soc. A211 (1952).

[20] B. Favier, F.S. Godeferd, and C. Cambon, Modeling the far-field acoustic emission of rotating turbulence, Journal of Turbulence 9 (2008), pp. 1-21.

[21] R.S. Rogallo Numerical experiments in homogeneous turbulence, NASA Tech. Mem. $81315,1981$. 
[22] R.H. Kraichnan, Diffusion by a random velocity field, Physics of Fluids 13 (1970), pp. $22-31$.

[23] D.J. Thomson and B.J. Devenish, Particle pair separation in kinematic simulations, J. Fluid Mech. 526 (2005), pp. 277-302.

[24] F. Nicolleau and G. Yu, Two-particle diffusion and locality assumption, Physics of Fluids 16 (2004), pp. 2309-2321.

[25] J. Dàvila and J.C. Vassilicos, Richardson's Pair Diffusion and the Stagnation Point Structure of Turbulence, Phys. Rev. Let. 91 (2003).

[26] J.C.H. Fung and J.C. Vassilicos, Two particles dispersion in turbulent-like flows, Phys. Rev. E. 57 (1998), pp. 1677-1690.

[27] M. Karweit et al., Simulation of the propagation of an acoustic wave through a turbulent velocity field, in 17th International Union of Theoretical and Applied Mechanics, Grenoble, France, 1988, pp. 21-27.

[28] W. Béchara et al., Stochastic approach to noise modeling for free turbulent flows, AIAA Journal 32 (1994), pp. 455-463.

[29] L. Liechtenstein, F.S. Godeferd, and C. Cambon, The role of nonlinearity in turbulent diffusion models for stably stratified and rotating turbulence, Int. J. of Heat and Fluid Flow 27(4) (2006), pp. 644-652.

[30] J.C.H. Fung et al., Kinematic simulation of homogeneous turbulence by unsteady random Fourier modes, J. Fluid Mech. 236 (1992), pp. 281-318.

[31] Y. Zhou and A.A. Praskovsky, A non-Gaussian phenomenological model for higherorder spectra in turbulence, Physics Letters A 178 (1993), pp. 138-142.

[32] M.A.I. Khan et al., Effects of LES sub-grid flow structure on particle deposition in a plane channel with a ribbed wall, Commun. Numer. Meth. Engng (2008). 
[33] S.A. Orszag and G.S. Patterson, Numerical simulation of three-dimensional homogeneous isotropic turbulence, Phys. Rev. Let. 28 (2) (1971), pp. 76-79.

[34] R. Rubinstein and Y. Zhou, Characterization of sound radiation by unresolved scales of motion in computational aeroacoustics, Eur. J. Mech. B/Fluids 21 (2002), pp. 105-111.

[35] G.W. He, R. Rubinstein, and L.P. Wang, Effects of subgrid-scale modeling on time correlations in large eddy simulation, Physics of Fluids 14 (2002), pp. 2186-2193.

[36] P. Flohr and J.C. Vassilicos, A scalar subgrid model with flow structure for large-eddy simulations of scalar variances, J. Fluid Mech. 407 (2000), pp. 315-349.

[37] H.D. Yao and G.W. He, A kinematic subgrid scale model for large eddy simulation of turbulence-generated sound, Journal of Turbulence 10 (2009), pp. 1-14.

[38] Y. Zhou, On the Lighthill relationship and sound generation from isotropic turbulence, Theoret. Comput. Fluid Dynamics 7 (1995), pp. 355-361.

[39] A. Witkowska and D. Juvé, Numerical study of noise from isotropic turbulence, J. of Comp. Acoustics 5 (1997), pp. 317-336.

[40] S. Sarkar and H. Hussaini, Computation of the sound generated by isotropic turbulence, ICASE Technical report 93-74 (1993). 


\begin{tabular}{|c|c|c|c|c|c|c|}
\hline \multicolumn{7}{|c|}{ DNS } \\
\hline Run & Resolution & Forcing & Realizations & \multicolumn{2}{|c|}{$R e_{\lambda}$} & $\Omega$ \\
\hline 1 & $256^{3}$ & $k \in[1: 4]$ & 3 & \multicolumn{2}{|c|}{156} & 0 \\
2 & $256^{3}$ & $k \in[1: 4]$ & 3 & \multicolumn{2}{|c|}{156} & 50 \\
\hline \hline \multicolumn{7}{|c|}{ KS } \\
\hline Run & Realizations & M & $\lambda^{\prime}$ & $\lambda$ & $\varpi_{n}$ & $\Omega$ \\
\hline 1 & 50 & 2000 & {$[0: 1]$} & 0.4 & $\sqrt{k_{n}^{3} E\left(k_{n}\right)}$ & 0 \\
2 & 50 & 2000 & {$[0.2: 1]$} & {$[0: 2]$} & $\sqrt{k_{n}^{3} E\left(k_{n}\right)}$ & 0 \\
3 & 100 & 2000 & 0.7 & 0.4 & $\sqrt{k_{n}^{3} E\left(k_{n}\right)}$ & 0 \\
4 & 100 & 2000 & 0.7 & 0.4 & $k_{n} u_{0}$ & 0 \\
5 & 1000 & 2000 & 0 & 0 & - & 10 \\
6 & 1000 & 2000 & 0.7 & 0.4 & $k_{n} u_{0}$ & 50 \\
\hline
\end{tabular}

Table 1: Numerical parameters of the results presented in section 3. $R e_{\lambda}$ is the Reynolds number based on the Taylor microscale at the beginning of the simulation. $M$ is the number of modes in KS. There is no such Cartesian grid in KS as in DNS since statistical quantities are computed directly in Fourier space.

\begin{tabular}{|c|c|c|c|c|c|c|c|}
\hline \multicolumn{2}{|c|}{ DNS } \\
\hline Run & Resolution & Forcing & Realizations & $R e_{\lambda}$ & \multicolumn{2}{|c|}{$\Omega$} \\
\hline 1 & $256^{3}$ & $k \in[1: 4]$ & 3 & 148 & \multicolumn{2}{|c|}{0} \\
2 & $256^{3}$ & $k \in[1: 4]$ & 3 & 148 & \multicolumn{2}{c|}{$[10: 50]$} \\
\hline \hline \multicolumn{7}{|c|}{ KS } \\
\hline Run & Resolution & Realizations & M & $\lambda^{\prime}$ & $\lambda$ & $\varpi_{n}$ & $\Omega$ \\
\hline 1 & $128^{3}$ & 30 & 2000 & 0.7 & 0.4 & $\sqrt{k_{n}^{3} E\left(k_{n}\right)}$ & 0 \\
2 & $128^{3}$ & 30 & 2000 & 0.7 & 0.4 & $k_{n} u_{0}$ & 0 \\
3 & $128^{3}$ & 10 & 1000 & 0 & 0 & - & {$[10: 50]$} \\
4 & $128^{3}$ & 30 & 2000 & 0.7 & 0.4 & $\sqrt{k_{n}^{3} E\left(k_{n}\right)}$ & 25 \\
5 & $128^{3}$ & 30 & 2000 & 0.7 & 0.4 & $k_{n} u_{0}$ & 25 \\
\hline
\end{tabular}

Table 2: Numerical parameters of the results presented in section 5. $R e_{\lambda}$ is the Reynolds number based on the Taylor microscale at the beginning of the simulation. $M$ is the number of modes in KS. 